\title{
OTOPALATODIGITAL SYNDROME TYPE I: NOVEL CHARACTERISTICS AND PRENATAL MANIFESTATIONS IN TWO SIBLINGS
}

\author{
Joksic $\mathrm{I}^{1, *}$, Cuturilo $\mathrm{G}^{2,3}$, Jurisic $\mathrm{A}^{1,2}$, Djuricic $\mathrm{S}^{4,5}$, Peterlin $\mathrm{B}^{6}$, Mijovic $\mathrm{M}^{2}$, \\ Karadzov Orlic $\mathrm{N}^{1,2}$, Egic $\mathrm{A}^{1,2}$, Milovanovic $\mathrm{Z}^{1,2}$ \\ *Corresponding Author: Ivana Joksic, M.D., Ph.D., Gynecology and Obstetrics Clinic "Narodni Front", \\ Kraljice Natalije 62, 11000 Belgrade, Serbia. Tel: +381-64-128-7643. Fax: +381-11-334-9651. E-mail: \\ ivanajoksic@yahoo.com
}

\begin{abstract}
Otopalatodigital spectrum disorder (OPDSD) is rare group of X-linked disorders caused by mutations in the filamin $\mathrm{A}(F L N A)$ gene. It is characterized by skeletal dysplasia of variable severity and different extra skeletal manifestations. Its presentation in the fetal period is quite unspecific, so diagnosis is usually made after birth. We present prenatal ultrasonography and postmortem findings that led us to a diagnosis of the mildest form of OPDSD (OPD type I) in two consecutive pregnancies. This is the first report on prenatal diagnosis (PND) of OPD type I. Affected fetuses showed facial dysmorphy (hypertelorism, micrognathia, cleft palate) and digital anomalies, features typical of OPD type I. In addition, microphtalmia and early neonatal death due to severe respiratory distress syndrome are described as a novel characteristics of the disorder. Clinical exome sequencing revealed a hemizygous missense pathogenic variant in the FLNA gene (NM_001110556.1: c.620C>T). We suggest that the presence of hypertelorism, micrognathia, digital anomalies on prenatal ultrasound examination should alert suspicion to OPDSD. Detailed clinical examination of mother and other female relatives is of great importance in establishing definitive diagnosis of OPD type I.
\end{abstract}

Keywords: Autopsy; Filamin A gene (FLNA), Microphtalmia; Otoplalatodigital syndrome type I (OPD type I); Prenatal diagnosis (PND).

\footnotetext{
"Gynecology and Obstetrics Clinic "Narodni Front", Belgrade, Serbia

${ }^{2}$ Faculty of Medicine, University of Belgrade, Belgrade, Serbia

${ }^{3}$ University Children's Hospital, Belgrade, Serbia

${ }^{4}$ Department of Pathology, Institute for Mother and Child Health Care "Dr Vukan Cupic", Belgrade, Serbia

${ }^{5}$ School of Medicine, University of Banjaluka, Banjaluka, Bosnia and Herzegovina

${ }^{6}$ Clinical Institute for Genomic Medicine, Ljubljana, Slovenia
}

\section{INTRODUCTION}

Otopalatodigital spectrum disorder (OPDSD) is a rare group of recognizable X-linked phenotypes caused by mutations in the filamin A (FLNA) gene [1]. It comprises four entities with overlapping clinical characteristics [otopalatodigital syndrome type I (OPD type I), OMIM 311300 , otopalatodigital syndrome type II (OPD type II), OMIM 304120; frontomethaphyseal dysplasia (FMD), OMIM 302620 and Melnick-Needles syndrome (MNS), OMIM 309350] [1,2]. The hallmark of these disorders are skeletal dysplasia of variable severity affecting both axial and appendicular skeleton accompanied with different extra skeletal anomalies [2]. The FLNA gene encodes cytoskeletal protein filamin A, that binds actin through its $\mathrm{N}$-terminal domain and other protein partners through the C-terminal domain [3]. It is expressed ubiquitously in cells and is required for membrane stability and linkage of signal transduction to the modulation of actin cytoskeleton [3]. Diagnosis of OPDSD is usually made postnatally, based on clinical characteristic and radiographic findings. Due to rather unspecific presentation in fetal period, diagnosis establishment based on ultrasonography abnormalities in the fetus is quite difficult, particularly in the mildest form OPD type I. So far, there are no published cases of prenatal diagnosis (PND) of OPD type I. We present prenatal ultrasonography and postmortem findings in two consecutive pregnancies of a young, non consanguineous couple that led us to the diagnosis of OPD type I.

\section{CASE REPORT}

Case 1. The second pregnancy of the couple was uneventful until the third trimester, when polyhidramnion, hypertelorsim and large gap between the toes were noticed. Quantitative fluorescence-polymerase chain reac- 
tion (QF-PCR) was performed at 36 weeks of gestation, and results were consistent with two copies of chromosomes 13, 18, 21 and male sex. Delivery was at term, birth weight was $2400 \mathrm{~g}$ (below P5), length $50 \mathrm{~cm}$ (P50), head circumference $35 \mathrm{~cm}$ (P25-50), Apgar score 8/8. At birth, multiple dysmorphic features were noted: widely opened fontanel, hypertelorism, downslanting palpebral fissures, broad nasal bridge, low set ears, mandibular hypoplasia, pointy chin, cleft secondary palate, pectus carinatum, and broad toes. Hypotonia was also present, and he required ventilator support. Eye examination revealed microphtalmia of the left eye. Postnatal imaging examinations showed normal structure of abdominal organs and heart. No additional anomalies were present. The karyotype was normal, male. Four days after birth, the baby died due to development of severe respiratory distress. Postmortem autopsy confirmed clinical findings, skeletal radiographs showed notably shortened and broadened phalanges, with duplication of distal phalanges of the thumbs.

Case 2. The subsequent (third) pregnancy of the couple was uneventful during the first trimester. Ultrasound examination at 22 weeks of gestation, showed normal fetal growth, however, broad nasal bridge, micrognathia and low set ears were detected (Figures 1 and 2). Fetal fingers were also broadly spaced with abducted thumb and possible agenesis of some metacarpal bones. Spina bifida in a lumbosacral region was also present (Figure 3). Fetal magnetic resonance imaging (MRI) was performed, confirming mandibular and nasal hypoplasia, as well as lumbosacral dysraphism with meningocele. Karyotyping yielded a normal male result. The pregnancy was terminated at 24

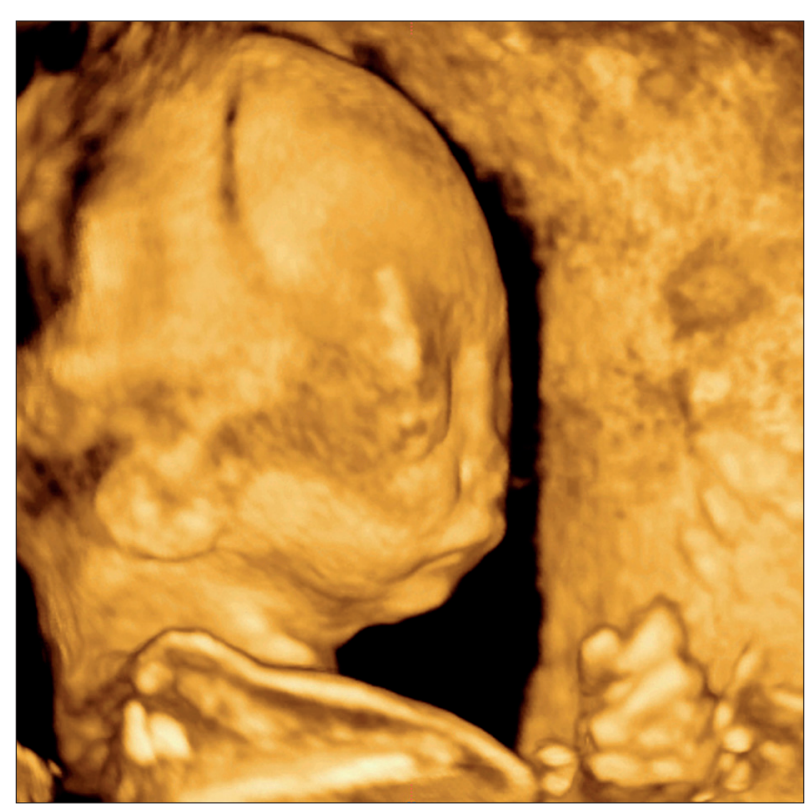

Figure 1. Ultrasound image of case 2 showing micrognathia and low-set ears. weeks of gestation at the parents request. Postmortem autopsy confirmed facial dysmorphism, hypertelorism, downslanting palpebral fissures and micrognathia. The index finger on both hands was significantly longer than the other fingers.

The parents were referred for genetic counseling. After clinical examination, it was noted that the mother had mild facial dysmorphism: hypertelorism, downslanting palpebral fissures, broad nasal bridge, flat facial profile,

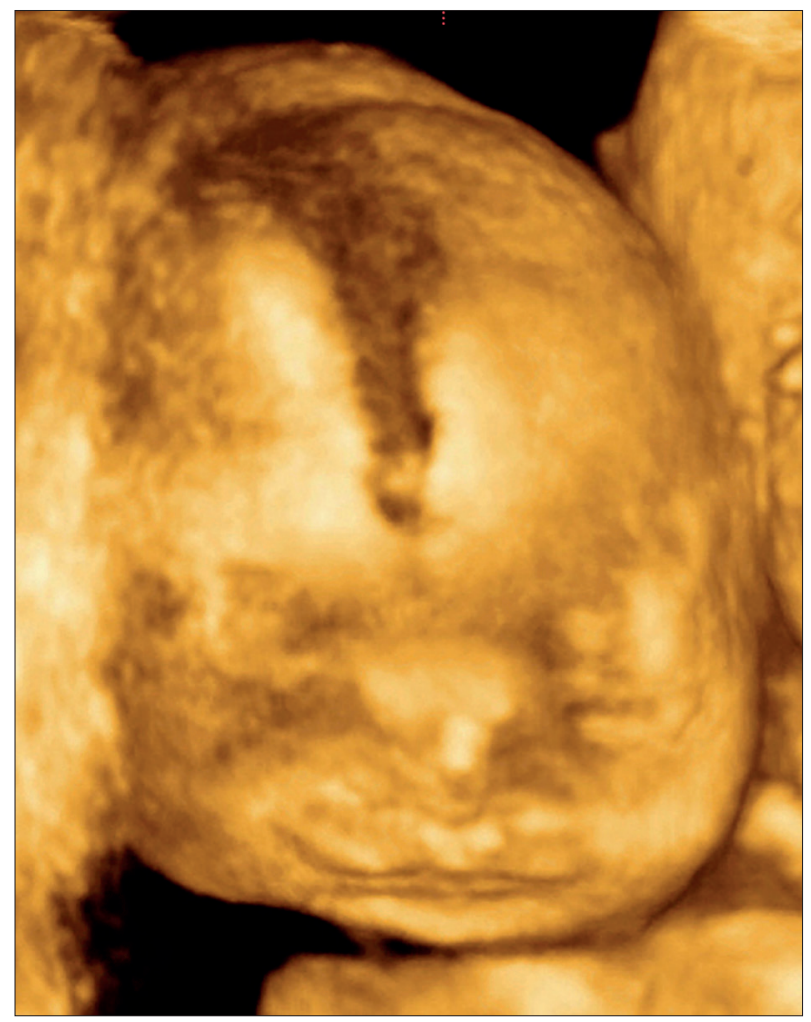

Figure 2. Ultrasound image of case 2 showing hypertelorism.

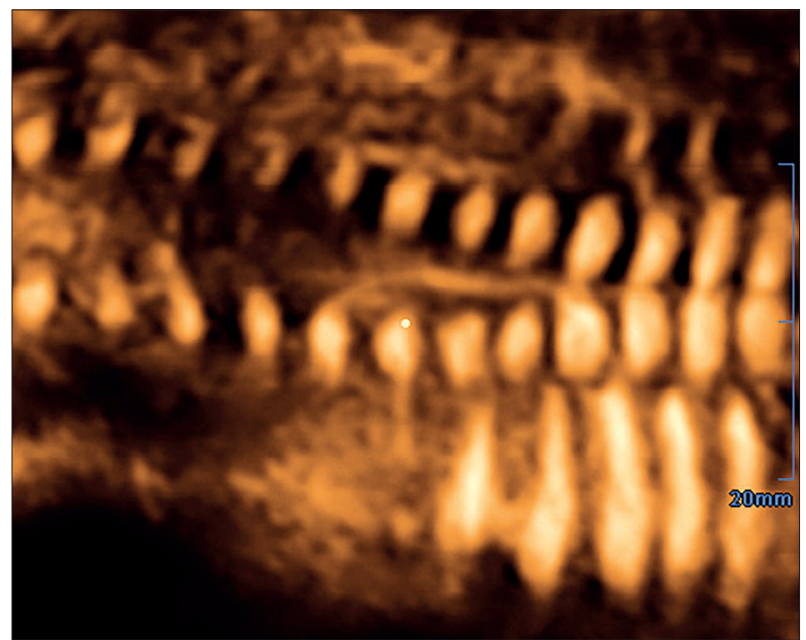

Figure 3. Ultrasound image of case 2 showing spinal dysraphism. 
prominent supraorbital ridges, pointy chin. She was otherwise healthy and of normal intelligence. In addition, we examined the daughter of the couple, and she showed similar facial dysmorphism as described in the mother. Neither mother nor daughter showed any additional skeletal changes.

The pattern of anomalies in more severely affected male offspring and dysmorphic features present in the mother and daughter have led us to the tentative diagnosis of OPDSD. Clinical exome sequencing followed by Sanger sequencing of the fetal DNA isolated from cord blood (case 2) revealed a hemizygous missense pathogenic gene variant in the FLNA gene (NM_001110556.1: c.620C > T), converting the codon for amino acid 207 from proline to leucine (NP 001104026.1: p.Pro207Leu, rs28935469), thus confirming OPDSD in the proband. In concordance with this, mother and daughter were found to be heterozygous carriers of the same mutation.

\section{DISCUSSION}

Mutations in the FLNA gene causative of OPDSD are usually missense mutations or small deletions that do not alter the reading frame, presenting a gain-of-functions mutation $[3,4]$. Filamin A binds F-actin through the Nterminal acting binding domain (ABD). The ABD is composed of two calponin homology domains, $\mathrm{CH} 1$ and $\mathrm{CH} 2$, which are highly conserved [3]. Mutations associated with OPD type I cluster exclusively in the $\mathrm{CH} 2$ domain of the protein, and it is considered that location of the mutation predicts severity of the disease [4]. Mutations in the $\mathrm{CH} 2$ domain tend to alter its activity and disturb interaction with F-actin, which is turn leads to cytoskeleton instability and disruption of signal transduction [3]. Although it has been shown that some mutations in $\mathrm{CH} 2$ (c.769G $>$ A) increase its affinity for F-actin, it is still not clear why chondrocyte function is primarily affected, while function of other cell types remains undisturbed $[2,3]$.

The mutation found in our patients is located in $\mathrm{CH} 2$ amino acid position 207, leading to a substitution of proline with leucine, and has previously been reported as pathogenic and causative of OPD type I. So far, three families with multiple affected males (total of seven individuals) carrying the c. $620 \mathrm{C}>\mathrm{T}$ mutation have been described in the literature [4-7]. Their clinical features are presented in Table 1. All affected individuals had facial dysmorphy (hypertelorism, downslating palpebral fissures), cleft palate, broad thumbs and great toes, chest wall deformities and deafness, features typical of OPD type I. Except for the presence of unilateral microphtalmia and early neonatal death in case 1 , the phenotypes of patients from our study is concordant with the clinical features described in other carriers of this mutation, which further supports genotypephenotype correlations in OPD type I. Although life expectancy is described as normal, and perinatal and neonatal mortality is not a feature of OPD type I, death of the boy (case 1) implies that respiratory complications with fatal outcome in early neonatal period can be expected even in the mildest form of OPDSD [1,2]. As a further support of possible respiratory complications in OPD type I, Robertson et al. [7], described a case of a boy who had required ventilator support until 4 months of age [7]. Ophtalomogic findings are rare in OPD type I. Congenital glaucoma and cataracts as well as corneal clouding have been described in OPD type II and MNS, but so far, microphtalmia has not been associated with any entity comprising OPDSD [8]. Microphtalmia can be caused by different environmental factors in early fetal development, such as infections, radiation or vitamin deficiency [8]. As the TORCH (toxoplasmosis, rubella, cytomegalovirus, herpes virus) analysis

Table 1. Clinical characteristics of OPD type I cases with the FLNA gene c.620C $>$ T pathologic variant.

\begin{tabular}{|l|c|c|c|c|c|c|c|c|c|}
\hline Parameters & Case 1 & Case 2a & $\begin{array}{c}\text { Case 3 } \\
{[7]}\end{array}$ & $\begin{array}{c}\text { Case 4 } \\
{[7]}\end{array}$ & $\begin{array}{c}\text { Case 5 } \\
{[6]}\end{array}$ & $\begin{array}{c}\text { Case 6 } \\
{[6]}\end{array}$ & $\begin{array}{c}\text { Case 7 } \\
{[6]}\end{array}$ & $\begin{array}{c}\text { Case 8 } \\
{[5]}\end{array}$ & $\begin{array}{c}\text { Case 9 } \\
{[\mathbf{5}]}\end{array}$ \\
\hline Hypertelorism & {$[+]$} & {$[+]$} & {$[+]$} & {$[+]$} & {$[+]$} & {$[+]$} & {$[+]$} & {$[+]$} & {$[+]$} \\
\hline Down-slanting fissures & {$[+]$} & {$[+]$} & {$[+]$} & {$[+]$} & {$[+]$} & {$[+]$} & {$[+]$} & {$[+]$} & {$[+]$} \\
\hline Cleft palate & {$[+]$} & {$[-]$} & {$[+]$} & {$[+]$} & {$[+]$} & {$[+]$} & {$[+]$} & {$[+]$} & {$[+]$} \\
\hline Micrognathia & {$[+]$} & {$[+]$} & {$[+]$} & {$[+]$} & {$[+]$} & {$[+]$} & {$[+]$} & {$[+]$} & {$[+]$} \\
\hline Deafness & $\mathrm{NA}$ & $\mathrm{NA}$ & {$[+]$} & {$[+]$} & {$[+]$} & {$[+]$} & {$[+]$} & $\mathrm{NA}$ & $\mathrm{NA}$ \\
\hline Digital anomalies & {$[+]$} & {$[+]$} & {$[+]$} & {$[+]$} & {$[+]$} & {$[+]$} & {$[+]$} & {$[+]$} & {$[+]$} \\
\hline Chest deformities & {$[+]$} & {$[-]$} & {$[-]$} & {$[+]$} & {$[+]$} & {$[+]$} & {$[+]$} & $\mathrm{NA}$ & $\mathrm{NA}$ \\
\hline Ophtalmologic findings & {$[+]$} & {$[-]$} & {$[-]$} & {$[-]$} & {$[-]$} & {$[-]$} & {$[-]$} & {$[-]$} & {$[-]$} \\
\hline Perinatal/neonatal mortality & {$[+]$} & $\mathrm{NA}$ & {$[-]$} & {$[-]$} & {$[-]$} & {$[-]$} & {$[-]$} & {$[-]$} & {$[-]$} \\
\hline
\end{tabular}

NA: not available; numbers in brackets indicate the cited references.

${ }^{a}$ Data for cases 1 and 2 are from this study. 
was negative in our patient and there were no indicators of vitamin deficiency or radiation exposure, we estimate that environmental factors are unlikely to be the cause of eye anomalies in our case. Thus, we propose microphtalmia as a possible new feature of OPDSD.

The phenotype of OPDSD seems to be poorly specific in fetal development. To date, only nine fetuses with an identified FLNA gene mutation have been reported, but none are associated with the OPD type I phenotype $[9,10]$. Ultrasonographic abnormalities detected in affected fetuses can vary greatly and differential diagnosis of potential disorders is very broad. All published prenatal cases with verified FLNA gene mutations had a more severe phenotype, OPD type II, MNS or FMD $[9,10]$. Even in cases with multiple skeletal and extra skeletal anomalies present, establishing a correct diagnosis is challenging due to significant clinical overlap between these entities. Filamin B related disorders, especially Larsen syndrome, have several phenotypic similarities with OPD type I, reflecting close homology with the FLNA gene [2]. Facial dysmorphism, cleft palate, short digits and long bones are common characteristic of OPD type I and Larsen syndrome, but mode of inheritance as well as presence of large joint dislocations differ in these two conditions. Oralfacial-digital syndrome is another possible differential diagnosis, considering the presence of telecantus, digital anomalies and cleft palate in this syndrome [9]. Prenatal diagnosis based on ultrasound examination is especially challenging in OPD type I, given the fact that sometimes only subtle skeletal malformations can be present. One of the dysmorphic syndromes that usually presents with similar but mild skeletal changes is Frank-ter Haar syndrome, caused by mutations of the $S H 3 P X D 2 B$ gene. However, its autosomal recessive mode of inheritance could be distinguished in some prenatal, familial cases [11]. In addition, patohistological examination might be necessary in order to make a more precise diagnosis as well as detailed examination of the parents and family members, who can also present with subtle signs of OPDSD. Next generation sequencing techniques are very valuable diagnostic tools in case of diagnosing a condition with a broad differential diagnosis such as OPDSD.

In conclusion, we suggest that the presence of hypertelorism, micrognathia, digital anomalies (wide spaced toes and broad thumbs) on prenatal ultrasound examination should alert suspicion to OPDSD. Detailed clinical examination of mother and other female relatives is of great importance in establishing definitive diagnosis of OPDSD. Microphtalmia and early neonatal death due to respiratory complications are suggested as a novel clinical features in OPD type I.
Declaration of Interest. The authors report no conflicts of interest. The authors alone are responsible for the content and writing of this article.

\section{REFERENCES}

1. Robertson SP. Otopalatodigital syndrome spectrum disorders: Otopalatodigital syndrome types 1 and 2, fronto-metaphyseal dysplasia and Melnick-Needles syndrome. Eur J Hum Genet. 2007; 15(1): 3-9.

2. Robertson S. In: Adam MP, Ardinger HH, Pagon RA, Wallace Se, Bean LJH, Stephens K, et al. (editors). X-Linked Otopalatodigital Spectrum Disorders (1993-2019). GeneReviews ${ }^{\circledR}$ [Internet]. Seattle, WA, USA: University of Washington, Seattle; 2005 [updated 2019].

3. Clark AR, Sawyer GM, Robertson SP, SutherlandSmith AJ. Skeletal dysplasias due to filamin A mutations result from a gain-of-function mechanism distinct from allelic neurological disorders. Hum Mol Genet. 2009; 18(24): 4791-4800.

4. Robertson S, Twigg S, Sutherland-Smith A, Biancalana V, Gorlin R, Horn D, et al.; The OPD Spectrum Disorders Clinical Collaborative Group. Localized mutations in the gene encoding the cytoskeletal protein filamin A cause diverse malformations in humans. Nat Genet. 2003; 33(4): 487-491.

5. Le Marec B, Odent S, Bracq E, Bulard MB, Bourdinière J, Babut JM. Oto-palato-digital type I syndrome in five generations. Relationship to the type II form. Ann Genet. 1988; 31(3): 155-161.

6. Dudding BA, Gorlin RJ, Langer LO. The oto-palatodigital syndrome. A new symptom-complex consisting of deafness, dwarfism, cleft palate, characteristic facies, and a generalized bone dysplasia. Am J Dis Child. 1967; 113(2): 214-221.

7. Robertson SP, Thompson S, Morgan T, Holder-Espinasse M, Martinot-Duquenoy V, Wilkie AO, et al. Postzygotic mutation and germline mosaicism in the oto-palatodigital syndrome spectrum disorders. Eur J Hum Genet. 2006; 14(5): 549-554.

8. Murphy-Ryan M, Babovic-Vuksanovic D, Lindor N. Bifid tongue, corneal clouding, and Dandy-Walker malformation in a male infant with otopalatodigital syndrome type 2. Am J Med Genet A. 2011; 155A(4): 855-859.

9. Naudion S, Moutton S, Coupry I, Sole G, Deforges $\mathrm{J}$, Guerineau E, et al. Fetal phenotypes in otopalatodigital spectrum disorders. Clin Genet. 2016; 89(3): 371-377. 
Joksic I, Cuturilo G, Jurisic A, Djuricic S, Peterlin B, Mijovic M, Karadzov Orlic N, Egic A, Milovanovic Z

10. Mariño-Enríquez A, Lapunzina P, Robertson SP, Rodríguez JI. Otopalatodigital syndrome type 2 in two siblings with a novel filamin A $629 \mathrm{G}>\mathrm{T}$ mutation: Clinical, pathological, and molecular findings. Am J Med Genet A. 2007; 143A(10):1120-1125.
11. Femitha P, Joy R, Gane BD, Adhisivan B, Bhat BV. Frank-ter Haar Syndrome in a newborn. Indian J Pediatr. 2012; 79(8): 1091-1093. 
